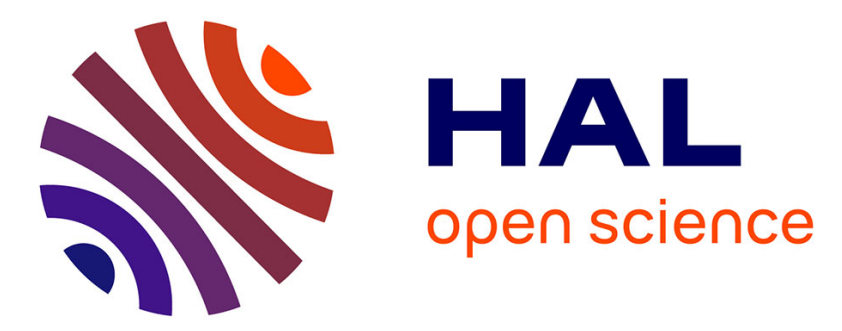

\title{
Seismic dispersion analysis feasibility for the subgrade investigation: measurement, experimental and numerical modeling
}

Donatienne Leparoux, Laurent Laguerre, Fabien Treyssede, Philippe Cote, Valéry Ferber

\section{To cite this version:}

Donatienne Leparoux, Laurent Laguerre, Fabien Treyssede, Philippe Cote, Valéry Ferber. Seismic dispersion analysis feasibility for the subgrade investigation: measurement, experimental and numerical modeling. Near Surface 2010 - 16th European Meeting of Environmental and Engineering Geophysics, Sep 2010, Zurich, Switzerland. 4p. hal-01064411

\section{HAL Id: hal-01064411 \\ https://hal.science/hal-01064411}

Submitted on 20 Feb 2015

HAL is a multi-disciplinary open access archive for the deposit and dissemination of scientific research documents, whether they are published or not. The documents may come from teaching and research institutions in France or abroad, or from public or private research centers.
L'archive ouverte pluridisciplinaire HAL, est destinée au dépôt et à la diffusion de documents scientifiques de niveau recherche, publiés ou non, émanant des établissements d'enseignement et de recherche français ou étrangers, des laboratoires publics ou privés. 


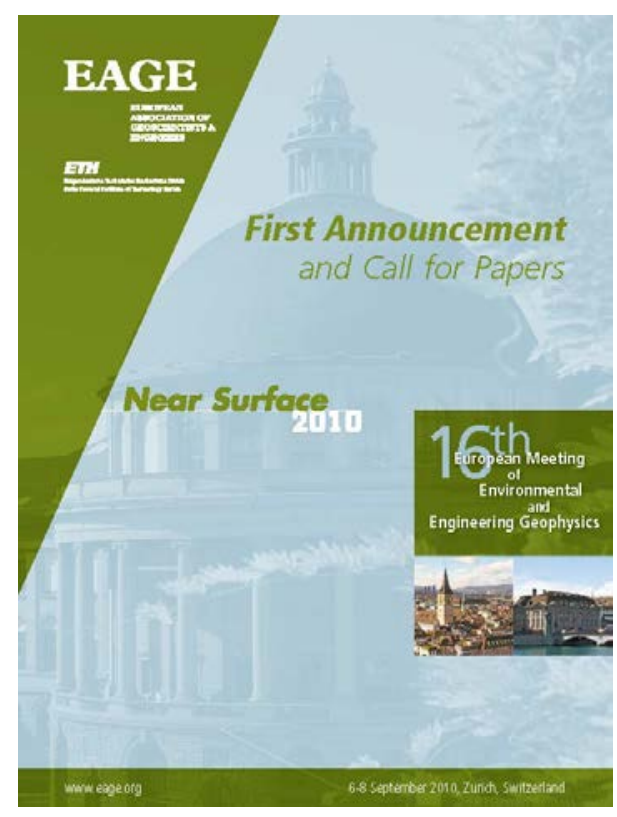

Seismic dispersion analysis feasibility for the subgrade investigation: measurement, experimental and numerical modeling

LEPAROUX, Donatienne ; LAGUERRE, Laurent ; TREYSSEDE, Fabien ; COTE, Philippe ; FERBER, Valéry 2010 


\section{Introduction}

In the construction of roads or railways, the capping layer is the last layer of the earthworks phase. This layer can be made of non bound aggregates or by a treatment of a soil with lime and/or hydraulic binder, such as cement or hydraulic road binders. In the latter case, the in situ testing of the capping layer performances should encompass treated soil modulus measurements, but sampling such materials is not often satisfactory, because of the risk of material degradation by the sampling itself. Consequently, a non destructive method, aiming at measuring the modulus of the treated materials, could be very useful. For this reason, we propose to study the feasibility of the seismic surface or guided waves dispersion analysis in order to recover the depth and the $\mathrm{S}$ wave velocity of the subgrade. Previous works provided results and analysis of the dispersion curves concerning the pavement auscultation (Ryden et al, 2004). However, in these cases, the subgrade was an underlying layer in the global zone of interest that includes the upper pavement layers where the measurement surface is the thin asphalt layer. In the present study, we focus on the subgrade layer in the case of under construction roads, before the shallower pavement layers are built because it should help to qualify the project acceptance concerning this earthworks phase. In this context, the issue deals with a two layers medium case where the investigated subgrade, whom the top is the measurement surface, lays above a low velocity zone, i.e. the natural soil. As described by Ryden et al. (2004), the resulting dispersion curves in the case of a high velocity upper layer should be typical of Lamb waves dispersion curves and could bring out higher modes that could be difficult to pick. In this case, they advocated the entire dispersion diagram inversion to avoid any subjective picking in the data (Ryden et al, 2006). However, the treated soil can contain heterogeneities unfavourable to the assumption of homogeneous layers presupposed to the dispersion diagram calculation. Thus a first feasibility stage, i.e. a field experimental data acquisition, was conducted to define the ability of seismic data to provide a coherent dispersion diagram in the spectral content required. The dispersion curve have been extracted and inverted with an iterative weighted least squares local minimization method (Hermann, 2002). In order to consider the possibility of inverting the entire dispersion diagram, a second feasibility stage consisted in analysing all the events that possibly occur in the dispersion diagram. For that, the measurement experience was reproduced at reduced scale in laboratory as a perfectly controlled experimental modelling approach. These data and more precisely the dispersion diagram is compared in one hand to the theoretical curves associated to the leakage attenuations and in an other hand to the theoretical dispersion diagram numerically calculated with an original method taking into account the source effects.

\section{In-situ experimental measurement}

Seismic measurements were carried out in "Lisle Pezou" (Loire et Cher, France) on a real underconstruction road. The subgrade is made up of clays with flints gravels that can be several centimetres size. It was a priori about $30 \mathrm{~cm}$ deep. Previous samples measurements provided seismic wave velocities estimation for defining the frequency range needed as well as the receiver spreading required which depend on the wavelength range needed. Data have been recorded by a high frequency system (10 MHz sampling) and a set of 30 accelerometers jointed with a rigid wood rod (figure 1). The receiver increment is $10 \mathrm{~cm}$. The seismic source was a small ball impact. Figure 2 shows the recorded data as a common shot gather setting where the data appear to be noisy due to the flint gravels that scattered $\mathrm{P}$ and guided waves.

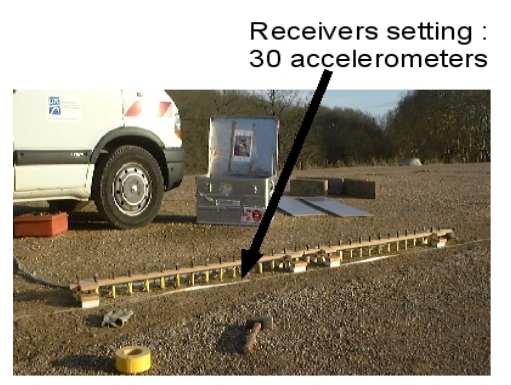

Figure 1 : field measurement setting

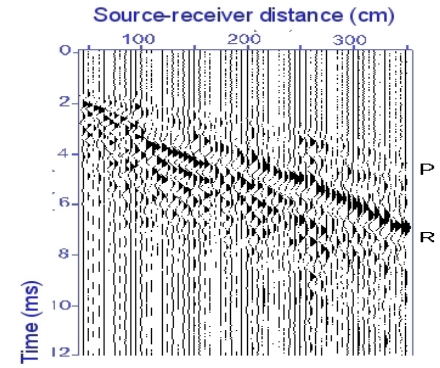

Figure 2 : one seismic shot gather 
Figure 3 shows the corresponding dispersion diagram calculated by the p- $\omega$ Transform (Park et al., 1998). In spite of the scattering effects visible in time data, it shows a continuous maximum amplitude curve in the $[350 \mathrm{~Hz}-1500 \mathrm{~Hz}]$ frequency range. The phase velocity equals to $450 \mathrm{~m} / \mathrm{s}$ for the lower frequencies and $550 \mathrm{~m} / \mathrm{s}$ for the higher frequencies (the corresponding wavelength are respectively $1.28 \mathrm{~m}$ and $0.36 \mathrm{~m}$ ). As expected, the curve presents the behaviors of the guided waves, i.e. a global shape closely to the Lamb waves $\mathrm{A}_{0}$ mode. However, the small contrast between the two layers authorizes to apply the local minimization method developed in Computer Programs in Seismology by Hermann, 2002, for inverting the extracted main dispersion curve. Figure (4) shows the inversion results for an initial homogeneous medium $(\mathrm{Vs}=800 \mathrm{~m} / \mathrm{s})$ and for which four layers are fixed that allowing velocities variations inside each layers : the resolution matrix (figure 4-b) presents a diagonal shape; the dispersion fit of the inverted model is in good agreement with data (figure 4-c); the inverted profile (figure 4-a) proposes a subgrade layer with $\mathrm{Vs}_{\mathrm{s}}=600 \mathrm{~m} / \mathrm{s}$ laying on the natural soil with Vs $\sim 50 \mathrm{~m} / \mathrm{s}$. This first results allow to validate the first stage of the feasibility concerning the subgrade investigation by surface waves : seismic waves generated by a small ball source are energetic enough to propagate along the required distances and the calculated dispersion diagram highlights a smooth curve that is able to be extracted and inverted in the suitable frequency range up to $1500 \mathrm{~Hz}$. In this context, the resulting inverted profile (figure 4-a) provides a global information about S-wave velocity in each layer. However, he tends toward a non uniform velocity in the upper layer (i.e. the investigated subgrade). Higher frequencies informations should be necessary to provide added informations concerning possible velocity variations with a better resolution. Actually the dispersion diagram (figure 3) seems to contain others modes (arrows 2 and 3) but these events are difficult to distinguish and they could also be generated by noisy scattering in the data. For these reasons, in order to better understand the different modes involved, we conducted an experimental modeling simulation of the field measurement through a Non Contact Laser Measurement Bench recently developed in LCPC (Bretaudeau et al, 2009).

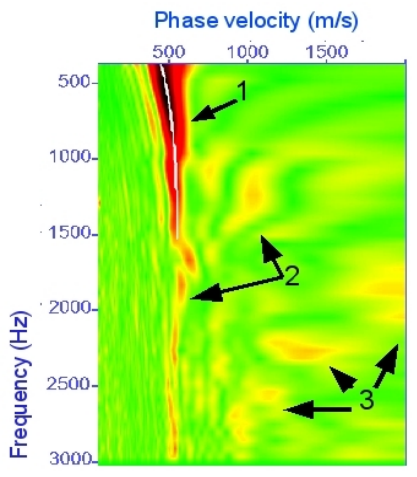

Figure 3 : dispersion diagram of field data
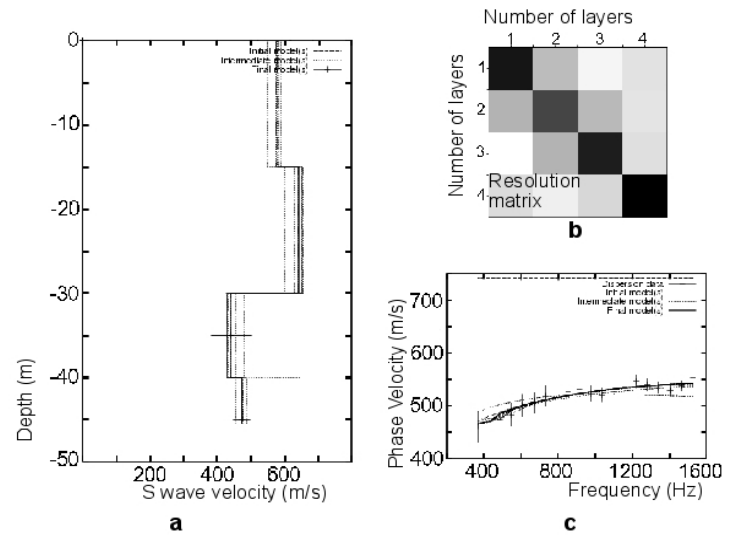

c

Figure 4 : inversion of the dispersion curve

from field data

\section{Small scale experimental measurement}

The experimental modeling was performed at reduced scale and controlled experimentally by an ultrasonic measurement bench (figure 5), called MUSC (for Mesures Ultrasonores Sans Contact in French), (Bretaudeau, 2009). Seismic data were collected at the medium surface by a Boss Nova heterodyne laser interferometer. The latter allows recording the absolute normal particle displacement without contact, avoiding disturbances caused by coupling. For our purpose, a piezoelectric source, coupled to the medium surface, emitted a $100 \mathrm{kHz}$ Ricker signal. The laser interferometer and the piezoelectric source were attached to two automated arms that could be moved over the model surface to a precision of $0.01 \mathrm{~mm}$. The receiver positions were spaced at $0.5 \mathrm{~mm}$. Sampling frequency was 10 $\mathrm{MHz}$ and data were recorded and coded on 16 bits. The two-layers model is made with epoxy resins with a scale ratio defined such as 1) the wavelength - to - layer thickness ratio at reduced scale is the same as at real scale ; 2) the Rayleigh waves velocities ratio between the two layers is similar in the 
model and in the real medium, within our means concerning the available resins. Seismic velocities in resins were measured on homogeneous samples. This led to a block composed of one layer $6.18 \mathrm{~mm}$ thick with the following characteristics : $\mathrm{Vr}=965 \mathrm{~m} / \mathrm{s}, \mathrm{Vp}=2300 \mathrm{~m} / \mathrm{s}, \mathrm{Vs}=1030 \mathrm{~m} / \mathrm{s}$, density $=1.3$ $\mathrm{g} / \mathrm{cm} 3$ above a second layer $100 \mathrm{~mm}$ thick with the following characteristics : $\mathrm{Vr}=625 \mathrm{~m} / \mathrm{s}, \mathrm{Vp}=1340$ $\mathrm{m} / \mathrm{s}, \mathrm{Vs}=670 \mathrm{~m} / \mathrm{s}$ density $=1.3 \mathrm{~g} / \mathrm{cm} 3$. Figure 6 shows the resulting dispersion diagram. The velocity axis has to be scaled by a factor equal to 0.57 and the frequency axis has to be scaled by a factor equal to 0.01 to be compared to the previous dispersion diagram previously presented on figure 3 . It highlights the main dispersion curve (arrow 1) from the low velocities at low frequencies to the high velocities at high frequencies similarly than in the real data (phase velocities are comparable after applying the scaling ratio above mentioned). Moreover, two other parts of curves are also clearly distinguishable (arrows 2 and 3 on figure 6). Theses experimental modeling will allow to analyses real but well controlled data that can be explained and analyzed in comparison to theoretical curves.

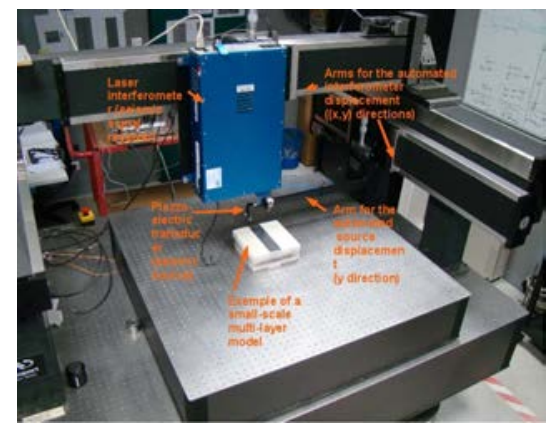

Figure 5 : Reduced scale laboratory MUSC for experimental modeling

\section{Theoretical dispersion curves and diagram.}

Dispersion curves for a two layers model (one layer over a semi-infinite half space) were derived with Disperse software (Lowe, 1995) based on a global matrix approach (Disperse, 2001). Properties of the layers are similar than the characteristics of the reduced scale model. Figure 8 shows the resulting phase velocity curves and the corresponding leakage attenuation up to $180 \mathrm{~dB} / \mathrm{m}$ respectively as a function of frequency up to $300 \mathrm{KHz}$ for comparison with experimental data. Arrows 1, 2 and 3 show the modes corresponding to the events visible on figure 6. Surprisingly, informations about the leakage attenuations would significate that the mode indicated by the arrow 3 should be very attenuated by leakage attenuations whereas it clearly appears on data (figure 6). The excitability effects (Ryden and Lowe, 2004) calculated by the same software cannot explain this phenomenon. We propose a new numerical approach for modeling the entire dispersion diagram that consists on 1) firstly calculating the guided modes with the Semi-Analytical Finite Element method (Lagasse et al. (1973) ; Datta et al. (1998)) ; and 2) secondly including source effects as following : the response to an excitation force is expanded as a sum of eigenvectors. Following the approach proposed by Liu and Achenbach (1995), modal coefficients can be calculated in a straightforward manner thanks to the use of an orthogonality relationship involving right and left eigenvectors.



Figure 6 : dispersion of Figure 7 : theoretical reduced scale data

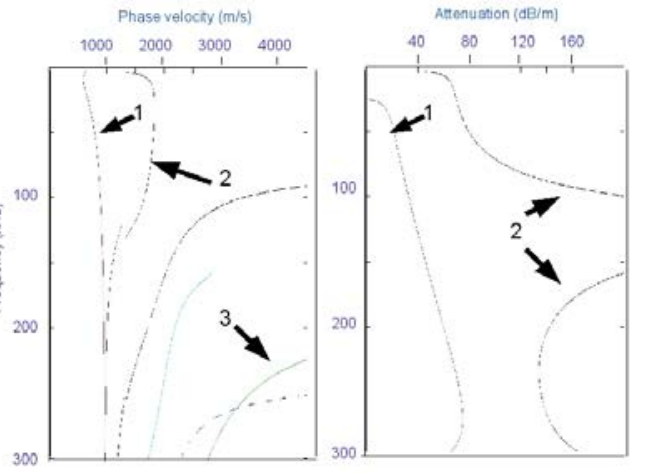

Figure 8: theoretical dispersion curves and leakage attenuation 
Figure 7 presents the theoretical dispersion diagram calculated by this principle. In this case, the three events indicated by the arrows are similar to those highlighted by the experimental dispersion diagram (figure 6). Comparisons with the field experimental dispersion diagram (figure 3) show similarities between the events indicated by arrows 1 and 2 respectively, taking into account of the scale ratios. However, arrow 3 seems to indicate not similar events in these two diagrams. This discrepancy could be due to the $\mathrm{P}$ waves velocity, because reduced scale model is only based on Rayleigh waves ratios. However, further numerical tests showed that modifying the $\mathrm{P}$ wave velocity in the upper layer also modifies this mode making it close to the field results.

\section{Conclusion and discussion}

The first feasibility stage involved field experimental measurements. The smooth main mode visible on the dispersion diagram was easy to be picked up to $1500 \mathrm{~Hz}$ and was able to be inverted to a $\mathrm{S}$ wave velocity profile leading to a global information concerning the subgrade. In a future aim to invert the entire dispersion diagram, the latter and more particularly other events not clearly distinguishable have been studied through a reduced scale experimental modeling providing a well controlled data set. The experimental diagram has been compared with theoretical results firstly calculated without any source effects and secondly by tacking into account the source effect through a new approach, showing a great similarity with reduced scale data and a partial similarity with field data. Discrepancies could be explained by experimental and numerical modeled $\mathrm{P}$ wave velocity unlike the real field one.

\section{Acknowledgements}

We thank Olivier Durand, Jacques Alexandre, Yasmina Boussafir for field measurements assistance.

\section{References}

Datta S. K., Shah A. H., Bratton R. L., Chakraborty T., 1998, Wave propagation in laminated composite plates, Journal of the Acoustical Society of America 83 (1988) 2020-2026.

Bretaudeau F., Leparoux D., Brossier R., and Abraham O., 2009, Small scale modeling, a tool to assess subsurface imaging methods - application to seismic full waveform inversion," in Proceedings of the 71st EAGE Conference and Exhibition, Amsterdam, 2009.

Disperse 2001 A system for generating dispersion curves. User's manual version 2.0.11. Guided ultrasonics, London.

Herrmann, 2002, Computer Programs in Seismology, Department of Earth and atmospheric Sciences of Saint Louis, GB.

Lagasse P.E., 1973, Higher-order finite element analysis of topographic guides supporting elastic surface waves, Journal of the Acoustical Society of America 53 (1973) 1116-1122.

Liu G.R., Achenbach J. D., 1995, Strip element method to analyse wave scattering by cracks in anisotropic laminated plates, Journal of Applied Mechanics, 62 (1995) 607-613.

Lowe M J S 1995 Matrix techniques for modelling ultrasonic waves in multilayered media IEEE Trans on Ultrasonics, Ferroelectrics and Freqjuency Control 42, 525-545

Park, C.B., Miller, R.D. and Xia, J., 1998, Imaging dispersion curves of surface waves on multichannel record. Soc. Exploration Geophys., New Orleans, Louisiana, USA.

Ryden N. and Lowe J. S., 2004, Guided wave propagation in three-layer pavement structures J. Acoust. Soc. Am. 116 (5), pp 2902-2913.

Ryden N., Park B. P., Ulriksen P., and Miller R.D. 2004, Multimodal Approach to Seismic Pavement Testing, Journal of geotechnical and geoenvironmental engineering.

Ryden N. and Park B. P., 2006, Fast simulated annealing inversion of surface waves on pavement using phase-velocity spectra, Geophysics, Vol71, N4, P R49-R58. 